\title{
Research on Model Reference Adaptive Hydraulic Servo Control System Based on Brain Emotional Learning Model
}

\author{
Yu Zhongan \\ Jiangxi University of Science and Technology \\ Ganzhou,China \\ yza999@126.com
}

\author{
Xie Zhenghua, Cao Yue \\ Jiangxi University of Science and Technology \\ Ganzhou,China
}

\begin{abstract}
According to the controlling of traditional complex hydraulic servo system is not precise enough, this paper presents an intelligent control method based on brain emotional learning (BEL) model. BEL model simulates the human brain emotional learning process, according to the neurobiology principle which has information organized function and emotional reaction between amygdale and orbital frontal cortex in the brain, established a calculating model (short for A-O model).This paper combined brain emotional learning model and model reference adaptive controlling, designed BEL intelligent controller, and made it (BELBIC) is used in hydraulic servo system. The results of the simulation have shown that BELBIC has fast speed reaction and good adaptive ability.
\end{abstract}

Keywords- rain emotional learning model, Reference model, Intelligent control, Hydraulic control system

\section{INTRODUCTION}

Emotion as an important part of human intelligence has caused more and more extensive concern, in the modern scientific research, the emotional control is become more and more important, it is not only a part of the ordinary study but also an important research branch.According to these neurobiology principle, Moren and others have proposed the calculating model of Brain Emotional Learning (BEL) based on neurophysiology in 2000. It simulates the information transfer model between amygdale and orbital frontal cortex (A-O) in the brain, constructed an emotional processing function model of general learning system.

At present, the hydraulic servo system, especially the electro-hydraulic servo system has occupied an important position in the military, technical aeronautics and automation area. Because of the hydraulic valves with the characteristics of dead zone and nonlinear in the hydraulic servo system, what is more, hydraulic system model will be changed under outside interference and running condition, so it brings great difficulty to the control system, and classical control theory is based on the accurate mathematical model, difficult to produce a good control effect to the complex and timevarying hydraulic servo system. The brain emotional learning model has advantages of simple calculation, adaptive ability and good robustness. Based on this, the paper introduced a brain emotional learning model, designed a model reference adaptive hydraulic servo controller based on brain emotional learning model, and a simulate experiment is carried out.

\section{BRAIN EMOTIONAL LEARNING (BEL) MODEL}

The brain emotional learning model is divided up into two parts, corresponding to orbital frontal cortex and amygdale, the amygdale part receive input signal from thalamus, and the orbital frontal cortex part receive input signal from cortex area and amygdale. As figure1 shows, each sensory input signal SIi corresponds to an almond node (thalamus stimulation also corresponds to a node).As the same, each sensory input signal SIi corresponds to an orbital frontal cortex node (hypothalamus node has no corresponding stimulate). All the outputs of the model through of a public output node $\mathrm{E}$, node $\mathrm{E}$ will simply accumulate the output of node $\mathrm{A}$, and then minus the output of node $\mathrm{O}$, making the amygdale and orbital frontal cortex outputs as the whole model output.

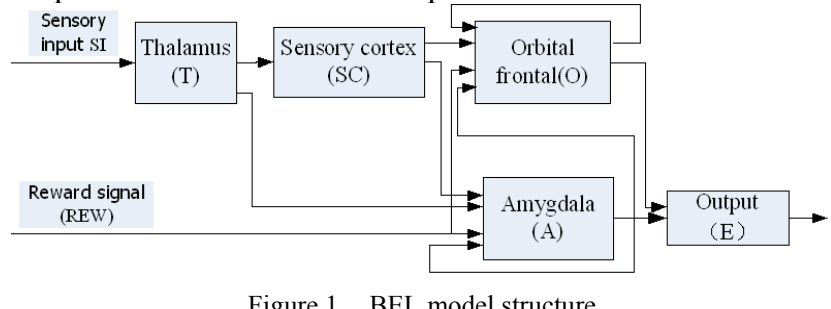

Figure 1. BEL model structure

The thalamus is connected with sensory input SI, its output is the maximum for all sensory signal SI, and as a part of the amygdale input:

$$
A_{\text {th }}=\max \left(S I_{i}\right)
$$

Every amygdale node has changes of continuous weight $\mathrm{V}$, any input values are multiplied by weight to get this node output values. Orbital frontal cortex node has a similar calculate way, which input values are multiplied by weight $\mathrm{W}$ to get node output values. Weight $\mathrm{Vi}$ is in proportion with poor value of Strengthen stimulation and amygdale node, the constant $\alpha$ adjust the speed of learning:

$$
\Delta V_{i}=\alpha\left(S I_{i} \max \left(0, R E W-\sum_{j} A_{i}\right)\right)
$$

The purpose of the orbital frontal part is to inhibit reaction when the response is not suitable. Therefore, the strengthened stimulate calculation of orbital frontal cortex node is the difference between previous output $\mathrm{E}$ and enhance signal REW. In other words, the values of the orbital frontal cortex node are got from compared expectation and received strengthening stimulation, if there are different, and then inhibit the output of model: 


$$
\Delta W_{i}=\beta\left(S I_{i} \sum_{j}\left(O_{j}-R E W\right)\right)
$$

The learning rules of orbital frontal cortex and amygdale are similar. The only and crucial difference is that the continuous weight of orbital frontal cortex can be increased or decreased in order to satisfy to track necessary constraints. Constant $\beta$ is another parameter of control study speed.

In conclusion, the amygdale and orbital frontal cortex node input/output calculation as follows:

$$
\begin{aligned}
& A_{i}=S I_{i} V_{i} ; \quad O_{i}=S I_{i} W_{i} \\
& E=\sum_{i} A_{i}-\sum_{i} O_{i}
\end{aligned}
$$

III. Model RefEREnCE Adaptive Hydraulic Servo CONTROL SYSTEM BASED ON BRAIN EMOTIONAL LEARNING MODEL

Combined model reference adaptive controlling and BEL model, established a model reference adaptive hydraulic servo control system based on brain emotional learning model (figure 2).

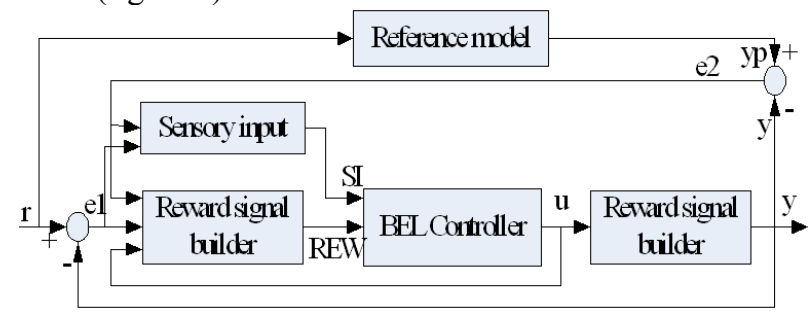

Figure 2. Adaptive hydraulic servo control system based on BEL model

Combine BEL model and actual system, make sure the function forms of sensory input signal SI and reward signal REW in advance, gone by the name of sensory input function and emotional hinted function respectively, they are mainly related with input /output, control quantity and tracking error factors of the system.BEL model will be introduced in hydraulic servo control system, and design a BEL controller, choose sensory input function and emotional hinted function, separately shows as following formulas (6) and (7):

$$
\begin{aligned}
& R E W=r_{1} e_{2}+r_{2} \int e_{2} d t+r_{3} \frac{d e_{2}}{d t}+r_{4} u+r_{5} S I \\
& S I=\left[\begin{array}{lll}
s_{1} e_{1} & s_{2} \int e_{1} d t \quad s_{3} \int e_{2} d t
\end{array}\right]
\end{aligned}
$$

Where e1、e2 are errors, $\int$ edt is integrated form for e,s1 、s2and s3、r1 to r5 are weight accommodation coefficient. Stimulate learning rewards signal REW is objective function, $\mathrm{r} 1$ to $\mathrm{r} 3$ are expectations of object error decreased, making the system output and reference model output to keep consistent as far as possible, $r 4$ is expectations of reduced output to the controller. Sensory input signal SI stimulate the A-O organization to study, by adjusting the weights of nodes in A-O organization, making the output signal of BEL model controller is consistent with reward signal.
BEL intelligent controller mot only have servo system error, integral and differential conditions, but also take the affective hinted function for target function, can regulate the weights of node in BEL model automatically according to the structure, therefore, it realize adaptive control of hydraulic servo system.

The following is the description of specific implementation steps of BEL model reference adaptive control algorithm:

(1)Initialized connected weights, set weight accommodation coefficient of sensory input function and emotional hinted function.

(2)Calculate sensory input signal SI and reward signal REW.

(3)Update connected weights of model.

(4)Calculate output signal $u$ of the BEL intelligent controller, output yp of reference model, output $y$ of hydraulic servo system.

(5)Judge whether the time has arrived, otherwise, return to step (2), then continue to run.

\section{SIMULATION AND EXPERIMENTAL ANALYSIS}

The mathematical model of hydraulic servo system for this paper is:

$$
G(s)=\frac{K_{g}}{\left(\frac{s}{398}+1\right)\left(\frac{s^{2}}{1574^{2}}+\frac{2 \zeta_{0}}{1574} s+1\right)}
$$

The damping ratio of the hydraulic system are $\zeta_{0}=0.5, \zeta_{0}=0.2, \zeta_{0}=0.8$, the basic parameters of BEL intelligent controller are set as:S1 $=0.43, \mathrm{~S} 2=0.35$, $\mathrm{r} 1=900, \mathrm{r} 2=30000, \mathrm{r} 3=1, \mathrm{r} 4=0$, the learning rate of weight are set as $\alpha=0.001, \beta=0.02$. In order to compared conveniently, considering the conventional control scheme based on the BEL model (without reference model,BELBIC), reference adaptive control method based on the PID model and the reference adaptive controller based on the BEL model, response simulation results were shown as figure 3 (a), figure 4 (a) and figure5 (a) of the three kinds of methods, we can come to the conclusion from the graph that:
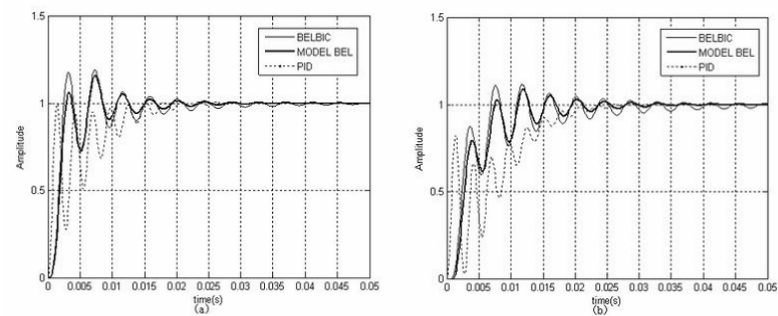

Figure 3. The comparison of the output response when each contro scheme damping ratio $\zeta_{0}=0.2$ 

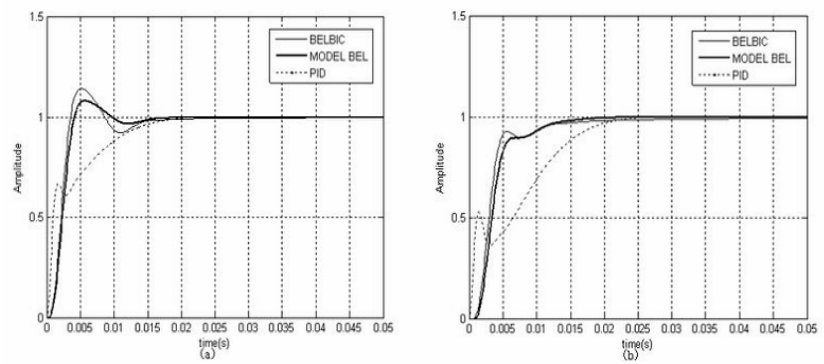

Figure 4. The comparison of the output response when each contro scheme damping ratio $\zeta_{0}=0.5$
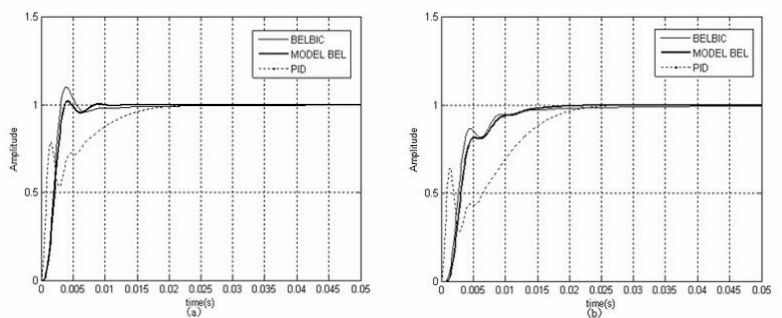

Figure 5. The comparison of the output response when each control

$$
\text { scheme damping ratio } \zeta_{0}=0.8
$$

(1) FIG. 3 (a), to adopt conventional BEL controller (BELBLC), system rise time is at $\mathrm{t}=0.0025 \mathrm{~S}$, the system output exist certain shocks, the system stable time is at $\mathrm{t}=0.0025 \mathrm{~S}$. To adopt PID model reference adaptive controller, system rise time is at $\mathrm{t}=0.0025 \mathrm{~S}$, after large vibration of response curve then reached stability is at $\mathrm{t}=0.023 \mathrm{~S}$. The reference model adaptive controller (MODEL BEL), based on the BEL model, the rise time of the system output curve is at $\mathrm{t}=0.003 \mathrm{~S}$, and arrived stable condition time is at $\mathrm{t}=0.02 \mathrm{~S}$.

(2) FIG. 4(a), to adopt conventional BEL controller (BELBLC), system rise time is at $\mathrm{t}=0.003 \mathrm{~S}$, the system output exist shocks, the system stable time is at $\mathrm{t}=0.015 \mathrm{~S}$. PID model reference adaptive controller, system rise time is at $\mathrm{t}=0.002 \mathrm{~S}$, and then reached stability is at $0.018 \mathrm{~s}$; The reference model adaptive controller (MODEL BEL), based on the BEL model, the rise time of the system is at $\mathrm{t}=0.0045 \mathrm{~S}$, and arrived stable condition at $\mathrm{t}=0.007 \mathrm{~S}$, have small vibration.

(3) FIG. 5(a), to adopt conventional BEL controller (BELBLC), system rise time is at $\mathrm{t}=0.0035 \mathrm{~S}$, the system stable time is at $\mathrm{t}=0.016 \mathrm{~S}$, the system output exist shocks. PID model reference adaptive controller, system rises slowly, and then reached stability at $\mathrm{t}=0.017 \mathrm{~S}$. The model reference adaptive controller (MODEL BEL), based on the BEL model, the rise time of the system is at $\mathrm{t}=0.004 \mathrm{~S}$, and arrive stable condition at $\mathrm{t}=0.016 \mathrm{~S}$, without steady-state error.

Give some disturbed signal to the hydraulic system, the parameters remain unchanged of each controller, the output response results of the three kinds of methods are shown as figure 3 (b), figure 4 (b) and FIG. 5 (b), so we can come to the conclusion from the graph that:
(1) FIG. 3 (b), under the disturbance, conventional BEL controller system rise time is at $\mathrm{t}=0.007 \mathrm{~S}$, the system stable time is at $\mathrm{t}=0.04 \mathrm{~S}$. To adopt PID model reference adaptive controller system, reached stability is at $\mathrm{t}=0.028 \mathrm{~S}$, with large oscillation amplitude. Under the controlling of reference adaptive controller based on the BEL model, system output response with small oscillation amplitude, and then reached stability at $\mathrm{t}=0.024 \mathrm{~S}$.

(2)FIG. 4 (b), under the disturbance, conventional BEL controller system stable time is at $\mathrm{t}=0.017 \mathrm{~S}$, without an overstrike. To adopt PID model reference adaptive controller system, reached stability is at $\mathrm{t}=0.015 \mathrm{~S}$, and the curve rise slowly. Under the controlled of reference adaptive controller based on the BEL model, system reached stability at $\mathrm{t}=0.015 \mathrm{~S}$, and the curve is smoothly.

(3)FIG. 5 (b), under the disturbance, conventional BEL controller system stable time is at $\mathrm{t}=0.02 \mathrm{~S}$, and the curve is smoothly. To adopt PID model reference adaptive controller system, reached stability is at $\mathrm{t}=0.022 \mathrm{~S}$, and the curve has certain fluctuations. Under the controlling of reference adaptive controller based on the BEL model, system reach stability at $\mathrm{t}=0.016 \mathrm{~S}$, and the curve is smoothly.

Contrast with the three control mode of figure 3, figure 4, figure 5 , we can come to the conclusion that the reference adaptive intelligent controller based on the BEL model has fastest response, strongest robustness, while based on the conventional control BEL controller take second place, and PID model reference adaptive controller has worst control effect.

\section{SUMMARIES}

In the traditional hydraulic servo system, introduced control strategy based on brain emotional learning model, established the reference adaptive control based on brain emotional learning model, designed BEL adaptive controller. Analysis the output response under these three control scheme system by contrast through experiments, indicated that the paper has proposed a method not only with good dynamic performance and robustness, but also can improve control precision.

\section{REFERENCES}

[1] J. Moren.Emotion and Learning-A Computational Model of the Amygdale[M], PhD dissertation, Lund University, 2002.

[2] J. Moren, C. Balkenius."A Computational Model of Emotional Learning in The Amygdale," From animals to animals 6: Proceedings of the 6th International conference on the simulation of adaptive behavior, Cambridge, Mass., 2000. The MIT Press.

[3] J.Sheikholeslami,D.Shahmirzadi,E.Semsar,C.Lucas,M.J.Yazdanpana h.ApplyingBrain Emotional Learning Algorithm for Multivariable Control of HVAC System[J].Journal of Intelligent \&Fuzzy Systems 17,2006.35-46

[4] .Babaie,R.Karimizandi.C.Lucas.Learning Based Brain Emotional Intelligence as A New Aspect for Development of An Alarm System[M].Springer-Verlag,2007.857-873

[5] iyang Zhen、 Daobo Wang、Zhisheng Wang、 Guoyong Huang, The turntable inverse compensating control based on brain emotional learning model[J]. Journal of Applied Science. 2009,3(3)

[6] ongqing Huang、Tianshi Li、Binggang Cao. Electro-hydraulic servo system with nonlinear adaptive control $[\mathrm{J}]$. Machine Tool and Hydraulics.1998,(03):7-8 